\title{
Sulfobetaine Polymers toward Application of Polydimethylsiloxane (PDMS) Surface Modification
}

\author{
Mutsuo Tanaka, ${ }^{1 *}$ Yoshikatsu Ogawa, ${ }^{2}$ Yoshiki Hirata, ${ }^{3}$ \\ Takahiro Sawaguchi, ${ }^{3}$ and Shigeru Kurosawa ${ }^{3 * *}$ \\ ${ }^{1}$ Department of Life Science \& Green Chemistry, Saitama Institute of Technology, \\ 1690 Fusaiji, Fukaya, Saitama 369-0293, Japan \\ ${ }^{2}$ Biomedical Research Institute, National Institute of Advanced Industrial Science and Technology (AIST), \\ 1-1-1 Higashi, Tsukuba, Ibaraki 305-8566, Japan \\ ${ }^{3}$ Health Research Institute, National Institute of Advanced Industrial Science and Technology (AIST), \\ 1-1-1 Higashi, Tsukuba, Ibaraki 305-8566, Japan
}

(Received June 29, 2018; accepted August 22, 2018)

Keywords: surface modification, sulfobetaine, polymer, polydimethylsiloxane

The surface modification of the polydimethylsiloxane (PDMS) elastomer was studied to obtain a durable hydrophilic surface leading to the reduction in surface tension with an aqueous solution for microflow channel application. Among hydrophilic polymers, it was found that a zwitterionic polymer, sulfobetaine, was adsorbed strongly on the PDMS surface treated with air plasma to afford a durable hydrophilic surface. The PDMS surface treated with air plasma is known to be a silica-like surface with negative charges, and electrostatic interaction, namely, polyion complex interaction between the sulfobetaine polymer and the treated PDMS surface, was sufficiently strong to maintain hydrophilicity even after heat treatment. Furthermore, cell culture experiments showed that the influence of the sulfobetaine polymer on cell growth was negligible, similar to 2-methacryloyloxyethyl phosphoryl choline (MPC) polymer, which is a common polymer for biomedical applications. The AFM observation of the PDMS surface modified with the sulfobetaine polymer suggests that the thickness of the sulfobetaine polymer film was very small on $\mathrm{nm}$ order; therefore, this modification method can maintain the microflow channel form. These results demonstrated that the sulfobetaine polymer could be a promising material for the surface modification of PDMS microflow channels for biomedical applications.

\section{Introduction}

Zwitterionic molecules have positive and negative charges in the same molecule and are well known as surface modification materials that afford biocompatible surfaces in selfassembled monolayers. ${ }^{(1-8)}$ On the other hand, the polymerization of zwitterionic molecules at the interface to form a biocompatible surface is investigated widely using a new polymerization method, atom transfer radical polymerization (ATRP). ${ }^{(9-14)}$ The surfaces fabricated by this

\footnotetext{
*Corresponding author: e-mail: mutsuo-tanaka@sit.ac.jp

** Corresponding author: e-mail: shigeru-kurosawa@aist.go.jp

https://doi.org/10.18494/SAM.2019.2039
} 
method are very attractive, especially in the biosensor field, because the surfaces modified with zwitterionic materials are highly resistant against the nonspecific adsorption of proteins and hydrophilic. ${ }^{(8,15-18)}$ Such surface properties are highly desirable to enhance the performance of biosensor systems. ${ }^{(19,20)}$ Therefore, various zwitterionic polymers have been synthesized and evaluated for that purpose, and phosphoryl choline and sulfobetaine groups are common as zwitterionic functional groups. Although ATRP is used to produce zwitterionic polymers, which are generally highly resistant against the nonspecific adsorption of proteins and are hydrophilic, to afford a polymer brush, ${ }^{(9-14)}$ the traditional coating method is also examined for surface modification. ${ }^{(12,21-24)}$

Recently, the application of the microflow channel for biosensor systems has been studied extensively as the microflow channel has the potential to enhance the performance of sensors. ${ }^{(25-27)}$ In the production of the microflow channel, polydimethylsiloxane (PDMS) is one of the useful materials, because it has good microfabrication properties for the microflow channel. However, there is a serious problem in the use of PDMS for the microflow channel, i.e., the PDMS surface is strongly hydrophobic, which disturbs the flow of aqueous samples owing to the significant surface tension at the narrow microflow channel. It was reported that the oxidation of the PDMS surface using plasma, corona discharge, and UV treatment afforded a silica-like layer and thus hydrophilicity, ${ }^{(28-34)}$ and many attempts of surface treatments to obtain a hydrophilic PDMS surface were conducted. Unfortunately, the PDMS surface recovers its hydrophobicity soon after the treatment because of the molecular mobility of PDMS, which is characteristic of elastomers. ${ }^{(28-32)}$ Only a few examples of surface modification using interfacial polymerization to afford a hydrophilic PDMS surface without recovering its hydrophobicity have been reported. ${ }^{(35,36)}$

In the coating method, it has been reported that electrostatic interaction plays a significant role in forming a durable modified surface, where the cationic polymers coated on the silica surface are adsorbed strongly owing to the electrostatic interaction ${ }^{(37,38)}$ with negative charges on the silica surface. ${ }^{(39)}$ The electrostatic interaction of polymers to form polyion complexes $^{(40-42)}$ is recognized as a useful method of obtaining a durable modified surface. The oxidized surface of PDMS is known to be silica-like with hydrophilicity, but the surface recovers its hydrophobicity soon after oxidation because of the molecular mobility of PDMS. ${ }^{(28-32)}$ As it is reported that the annealing treatment of the coating surface with polymers enhances electrostatic interaction through the structural arrangement of polymers due to their flexibility, ${ }^{(43)}$ we expected that zwitterionic polymers might induce a strong electrostatic interaction with the oxidized PDMS surface similar to cationic polymers through structural arrangement. Studies revealed that zwitterionic polymers are adsorbed on the PDMS surface treated with air plasma to afford a hydrophilic surface, and the adsorption of zwitterionic polymers on the PDMS surface is sufficiently strong to resist ultrasonic cleaning in water. ${ }^{(44,45)}$ The hydrophilicity of the PDMS surface was maintained even 1 month after the modification, while the PDMS surface treated only with air plasma recovered its hydrophobicity within four days owing to the molecular mobility of PDMS as reported previously. ${ }^{(28-34)}$ We compared two heat-treated zwitterionic polymers, sulfobetaine (C3SB) and phosphoryl choline (MPC) (Fig. 1), and C3SB afforded a more durable hydrophilic surface than MPC. ${ }^{(45)}$ It has been reported 
that the sulfobetaine moiety is cation-like, but the phosphoryl choline moiety is anion-like. ${ }^{(46)}$ Therefore, it is considered that the negatively charged silica-like surface of PDMS treated with air plasma interacts with the sulfobetaine polymer more strongly than with the MPC polymer. Furthermore, we adopted the amide bond to combine the sulfobetaine moiety with the methacryl moiety in the molecular design of the sulfobetaine monomer, because the amide bond resists the nonspecific adsorption of proteins ${ }^{(47)}$ and hydrolyze more effectively than the ester bond under common conditions.

In control experiments, the PDMS surface modified with a monomer solution of C3SB by the same procedure recovered its hydrophobicity soon after the modification. Therefore, it is evident that the adsorption of $\mathrm{C} 3 \mathrm{SB}$ on the treated PDMS surface is derived from the polymer effect to form a polyion complex, and the electrostatic interaction is sufficiently strong to suppress the molecular mobility of PDMS leading to a durable hydrophilic surface as illustrated in Fig. 2. Furthermore, C3SB with a higher molecular weight suppressed the molecular mobility of PDMS more effectively to afford a more durable hydrophilic PDMS surface. This result reflects that the electrostatic interaction induced by the polyion complex formation depends on the molecular weight of the polymers.

In this work, we studied the synthesis of the sulfobetaine polymer with a high molecular weight and its cell toxicity for biomedical applications.

\section{Materials and Methods}

\subsection{General descriptions of materials used}

All chemicals were commercially available and used as received without additional purification. The MPC monomer was purchased and used as received. All aqueous solutions

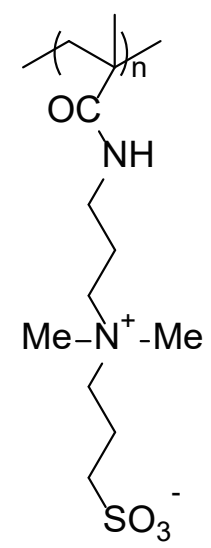

C3SB

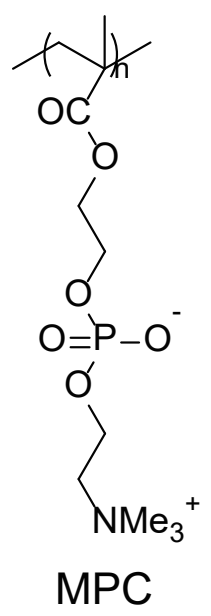

Fig. 1. Compared zwitterionic polymers.

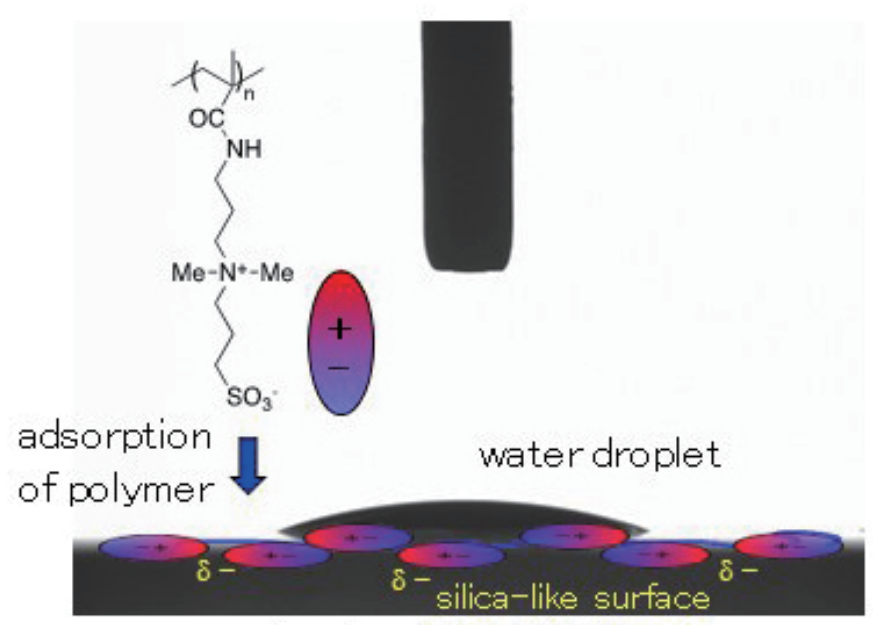

hydrophilic PDMS

Fig. 2. (Color online) Schematic diagram of polyion complex on PDMS. 
were prepared using pure water obtained from a Millipore system. The PDMS used in this work was a silicone rubber sheet (AS ONE $1.0 \mathrm{t} \times 30 \times 10 \mathrm{~mm}$ ). A plasma device, YHS-R (Sakigake Semiconductor Ltd.), was used for the surface treatment of PDMS.

\subsection{General polymerization procedures with ammonium persulfate}

To a flask, the C3SB monomer (1.46 g, $5 \mathrm{mmol})$, ammonium persulfate (11.4 $\mathrm{mg}$, $0.05 \mathrm{mmol})$, and water $(5 \mathrm{~mL})$ were added, and the flask was sealed with aluminum foil without deoxygenation. The reaction mixture was warmed and allowed to stand at $50{ }^{\circ} \mathrm{C}$ for $15 \mathrm{~h}$. To the reaction mixture, water $(5 \mathrm{~mL})$ was added, and the reaction mixture was stirred. The reaction mixture was poured into methanol $(50 \mathrm{~mL})$ with vigorous stirring, and the obtained solution was allowed to stand overnight. The polymer obtained as the precipitate was collected and rinsed with methanol. After drying in vacuum, the polymer was obtained with quantitative yield as a colorless solid.

\subsection{Surface modification of PDMS}

The PDMS surface was treated with air plasma before surface modification. The air-plasma irradiation conditions were output, $100 \mathrm{~W}$; pressure, $50 \mathrm{~Pa}$; air flow, $100 \mathrm{~mL} / \mathrm{min}$; irradiation period, 3 min. After the air-plasma treatment, PDMS was immersed in an aqueous solution of polymers $(1 \mathrm{mg} / \mathrm{mL})$ for $10 \mathrm{~min}$. PDMS was rinsed for 1 min under sonication and dried with nitrogen blowing.

\subsection{Cell culture and influence of zwitterionic polymers on cell growth}

The human cervical carcinoma cell line HeLa was provided by RIKEN BRC through the National Bio-Resource Project of MEXT, Japan. G418 solution was purchased from Thermo Fisher Scientific (NY, USA). HeLa cells were cultured in a minimum essential medium (MEM) (Thermo Fisher Scientific, NY, USA) supplemented with 10\% fetal bovine serum (FBS) at 37 ${ }^{\circ} \mathrm{C}$ under a humidified $5 \% \mathrm{CO}_{2}$ atmosphere. The influence of zwitterionic polymers on cell growth was studied as follows. HeLa cells $\left(2 \times 10^{3} /\right.$ well $)$ were seeded in 96 -well plates and incubated for $24 \mathrm{~h}$. After that, a fresh medium containing a zwitterionic polymer $(0,0.1,0.2$, $0.3,0.4,0.5 \mathrm{mg} / \mathrm{mL}$ ) was added to replace the culture medium. After incubation for $48 \mathrm{~h}$, cell viability was measured using Cell Counting Kit-8 (Dojindo, Kumamoto, Japan) in accordance with the instructions provided by the manufacturer.

\subsection{Atomic force microscopy (AFM) analysis of PDMS surface}

AFM images were collected using a NanoScope IIIa Multimode AFM System (Veeco Instruments Inc., Santa Barbara, CA) in the tapping mode under ambient conditions. Silicon cantilevers with a nominal resonance frequency of $300-400 \mathrm{kHz}$, a force constant of $\sim 120$ $\mathrm{N} / \mathrm{m}$, and a tip radius of $<10 \mathrm{~nm}$ (ppp-NCH-AuD, Nano World Holding AG, Schaffhausen, 
Switzerland) were used in the AFM experiments. Images were analyzed using the software provided by the manufacturer.

\section{Results and Discussion}

\subsection{Polymer synthesis}

As C3SB with a higher molecular weight afforded a more durable hydrophilic PDMS surface in the previous work, we examined the synthesis conditions to obtain C3SB with a higher molecular weight. In the case of a common polymerization method using azobisisobutyronitrile (AIBN), it is well known that the molecular weight of the obtained polymer increases with the increase in monomer concentration or with the decrease in AIBN concentration. Therefore, polymerization was carried out with various monomer and AIBN concentrations. When the monomer concentration was $167 \mathrm{mM}$ in methanol, the molecular weight of the obtained polymer with various AIBN concentrations between 1.67 and $8.33 \mathrm{mM}$ was almost constant, that is, 30000-40000. Attempts to increase the monomer concentration failed because of the low solubility of the monomer in methanol. Therefore, water, in which the monomer has a higher solubility, for the monomer, was adopted as a solvent. When the monomer concentration was increased to $1 \mathrm{M}$, the molecular weight of C3SB reached 79000. However, the reaction mixture was heterogeneous again because of the low solubility of AIBN in water. Instead of AIBN, various water-soluble polymerization initiators, 2,2'-azobis[2-(2-imidazolin-2-yl) propane] dihydrochloride (AIP), 2,2'-azobis(2-methylpropionamidine) dihydrochloride (AMPA), and ammonium persulfate (APS), were evaluated using $1 \mathrm{M}$ monomer solution with a $10 \mathrm{mM}$ initiator. Interestingly, these initiators could work well without deoxygenation; therefore, the polymerization was examined not only under deoxygenation condition but also under atmospheric condition. The initiator structures and polymerization results are summarized in Fig. 3 and Table 1, respectively. Although the polymerization proceeded slowly under atmospheric condition, there was no meaningful difference in the molecular weight of the obtained polymers. Among these initiators, ammonium persulfate afforded a larger molecular weight with lower yields. As ammonium persulfate is inexpensive and a common reagent for acrylamide polymerization in the molecular biology field, we used it as the polymerization initiator, taking into account its biomedical applications.

Using various ammonium persulfate concentrations, polymerization was conducted and the molecular weight of the obtained C3SB polymer was examined. The results are shown in Table 2.
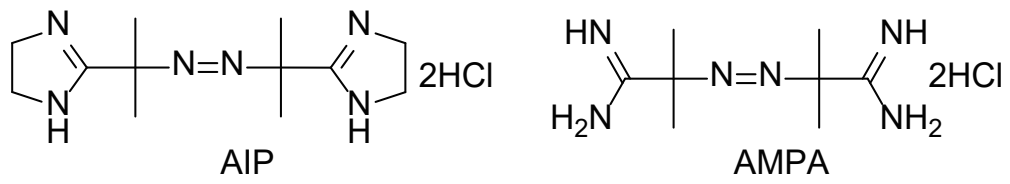

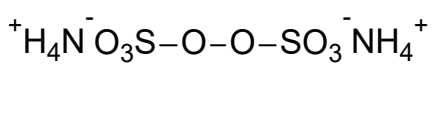

Fig. 3. Water-soluble polymerization initiator. 
Table 1

Synthesis of C3SB with various initiators.

\begin{tabular}{lccc}
\hline Initiator & Condition & Yield (\%) & Molecular weight \\
\hline \multirow{2}{*}{ AIP } & deoxygenation & quantitative & 69000 \\
& atmosphere & 96 & 67000 \\
\hline \multirow{2}{*}{ AMPA } & deoxygenation & quantitative & 72000 \\
& atmosphere & 92 & 71000 \\
\hline \multirow{2}{*}{ APS } & deoxygenation & 83 & 93000 \\
& atmosphere & 54 & 89000 \\
\hline
\end{tabular}

Polymerization was carried out at $50{ }^{\circ} \mathrm{C}$ for $3 \mathrm{~h}$ using a $1 \mathrm{M}$ monomer solution.

Table 2

Synthesis of C3SB with ammonium persulfate.

\begin{tabular}{lccc}
\hline $\begin{array}{l}\text { Initiator concentration } \\
(\mathrm{mM})\end{array}$ & Condition & Yield (\%) & Molecular weight \\
\hline \multirow{2}{*}{10} & deoxygenation & 95 & 88000 \\
& atmospheric & quantitative & 84000 \\
\hline \multirow{2}{*}{2} & deoxygenation & 97 & 80000 \\
& atmosphere & 85 & 79000 \\
\hline & deoxygenation & 98 & 76000 \\
& atmosphere & 76 & 74000 \\
\hline
\end{tabular}

Polymerization was carried out at $50{ }^{\circ} \mathrm{C}$ for $15 \mathrm{~h}$.

Again, no significant difference depending on the polymerization conditions was observed in the molecular weight of the polymers, being between 70000 and 90000 . The C3SB polymer with 84000 molecular weight was obtained quantitatively by reacting 1 M C3SB monomer aqueous solution with $10 \mathrm{mM}$ ammonium persulfate at $50{ }^{\circ} \mathrm{C}$ for $15 \mathrm{~h}$ without deoxygenation, and the synthesis procedure is very simple and preferable for application.

We also examined the polymerization with ATRP and reversible addition/fragmentation chain transfer (RAFT) methods. However, attempts to obtain C3SB by ATRP failed without polymerization at all. In the case of RAFT, polymerization occurred, but the molecular weight of the obtained C3SB was low, being 25000, and the molecular weight distribution was no more than around 5.

\subsection{Cell toxicity of polymers}

Although our previous works on $\mathrm{C} 3 \mathrm{SB}$ and $\mathrm{MPC}$ suggested that $\mathrm{C} 3 \mathrm{SB}$ is superior to $\mathrm{MPC}$ in terms of the durability of hydrophilicity, ${ }^{(44,45)}$ the cell toxicity of the phosphoryl choline polymer is known to be quite low and considered to be a promising material for biomedical applications. Therefore, we evaluated the cell toxicity of C3SB in comparison with that of MPC in terms of the influence of the polymers on cell growth according to the reported procedures. ${ }^{(48,49)}$ We adopted the human cervical carcinoma cell line HeLa. An aminoglycoside antibiotic, G418, was also used for the positive control experiment as it is known to inhibit the growth of HeLa cells. The C3SB polymer was synthesized by reacting with $1 \mathrm{M} \mathrm{C} 3 \mathrm{SB}$ monomer aqueous solution with $10 \mathrm{mM}$ ammonium persulfate at $50{ }^{\circ} \mathrm{C}$ for $15 \mathrm{~h}$ without deoxygenation, while MPC was synthesized according to the procedure reported in Ref. 44. The molecular weights 
of C3SB and MPC are 80000 and 67000, respectively. The results are summarized in Fig. 4. The vertical axis shows cell viability, namely, relative cell number percentage compared with the negative control experiment in the absence of polymers and G418, and the values are shown as means \pm standard deviations $(n=5)$. On the other hand, the horizontal axis indicates the concentrations of C3SB, MPC, and G418 in the culture media. In Fig. 4, there is no meaningful difference between C3SB and MPC in cell viability, being almost 100\% regardless of the polymer concentration, while the cell viability decreased significantly with the increase in G418 concentration. This result clearly demonstrates that the cell toxicity of C3SB is low similarly to that of MPC, and C3SB could also have a potential as a material for biomedical applications.

\subsection{Surface observation by AFM}

The surface roughness of PDMS was examined in detail by AFM observation because surface roughness is known to influence significantly the hydrophilicity. The AFM images of PDMS surfaces, the intact surface, the surface with air-plasma treatment, and the surface modified with C3SB are shown in $10 \times 10 \mu \mathrm{m}^{2}$ and $1 \times 1 \mu \mathrm{m}^{2}$ scans in Fig. 5. All AFM images showed a significant wavy production notch with a granular structure. The surface roughnesses $(R a)$ of the intact surface of PDMS were 14.5 and $1.30 \mathrm{~nm}$ in $10 \times 10 \mu \mathrm{m}^{2}$ and $1 \times 1 \mu \mathrm{m}^{2}$ scans, respectively. After air-plasma treatment, Ra was $14.5 \mathrm{~nm}$ in the $10 \times 10 \mu \mathrm{m}^{2}$ scan, but it decreased to $0.64 \mathrm{~nm}$ in the case of the $1 \times 1 \mu \mathrm{m}^{2}$ scan. This significant decrease in $R a$ with the $1 \times 1 \mu \mathrm{m}^{2}$ scan suggests that the PDMS surface is smoothened by air-plasma treatment. By surface modification with $\mathrm{C} 3 \mathrm{SB}$, both surface roughnesses were drastically decreased to 3.12 and $0.39 \mathrm{~nm}$ in the $10 \times 10$ and $1 \times 1 \mu \mathrm{m}^{2}$ scans, respectively. In the AFM images, the wavy production notch seems to be filled with $\mathrm{C} 3 \mathrm{SB}$, and the granular structure clearly became flatter, resulting in a clear reduction in $R a$. Therefore, it is obvious that the hydrophilicity of the modified PDMS surface reflects the properties of the zwitterionic polymers adsorbed on

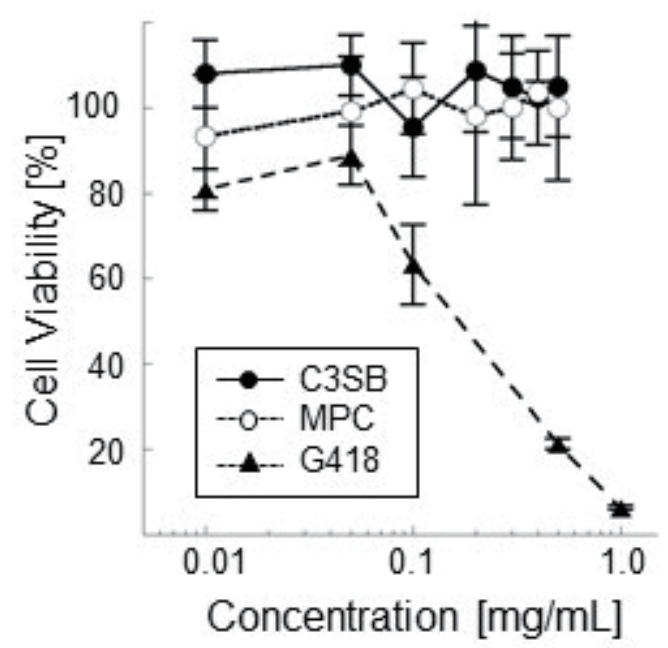

Fig. 4. Cell growth with polymers. 


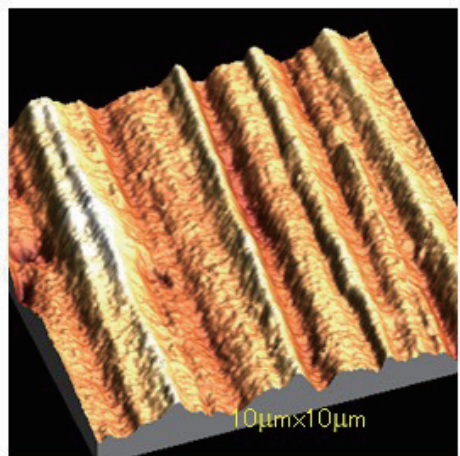

$\mathrm{Ra}=14.5 \mathrm{~nm}$

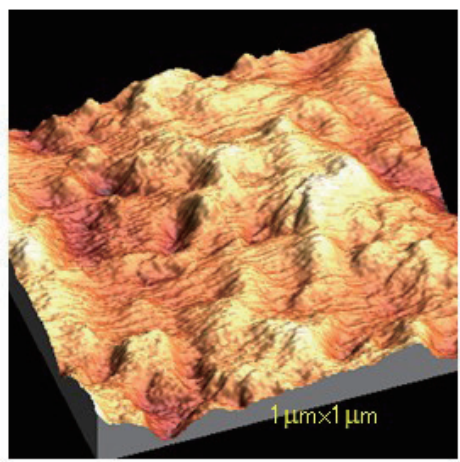

$\mathrm{Ra}=1.30 \mathrm{~nm}$

intact surface

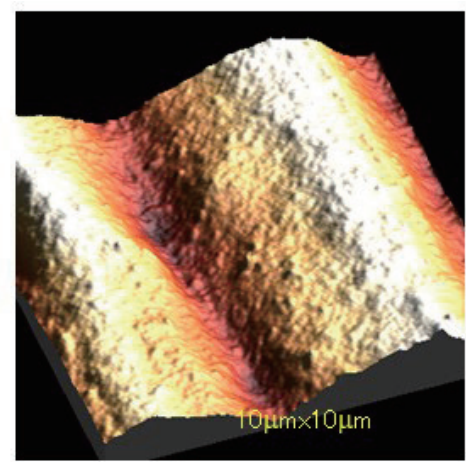

$\mathrm{Ra}=14.5 \mathrm{~nm}$

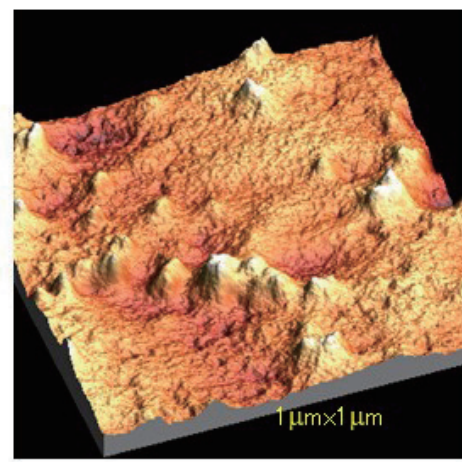

$\mathrm{Ra}=0.64 \mathrm{~nm}$

after plasma treatment

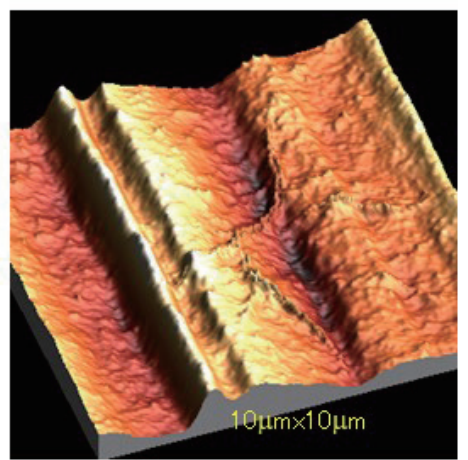

$\mathrm{Ra}=3.12 \mathrm{~nm}$

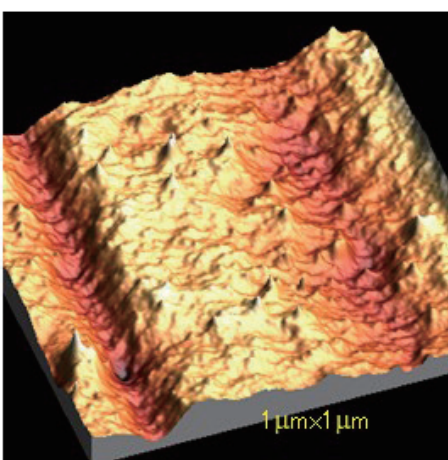

$\mathrm{Ra}=0.39 \mathrm{~nm}$ after C3SB modification

Fig. 5. (Color online) AFM images of PDMS surfaces.

the PDMS surface but not the surface roughness of PDMS. Furthermore, the PDMS surface covered with C3SB became smooth, but the structures are still clearly recognizable. These AFM observations suggest that the thickness of C3SB films is very small on nanometer order. Therefore, this modification method has potential applications to the surface modification of microflow channels as this method can maintain the microflow channel form after modification compared with other coating methods.

\section{Conclusions}

It was found that the C3SB polymer with 70000-90000 molecular weight was obtained quantitatively by reacting with $1 \mathrm{M} \mathrm{C} 3 \mathrm{SB}$ monomer aqueous solution with $10 \mathrm{mM}$ ammonium persulfate at $50{ }^{\circ} \mathrm{C}$ for $15 \mathrm{~h}$ without deoxygenation, and the synthesis procedure is very simple and preferable for application. The cell toxicity of the sulfobetaine polymer is as low as that of the MPC polymer in terms of cell growth according to the cell culture experiments. Furthermore, the AFM observation of the PDMS surface modified with C3SB showed that the thickness of the C3SB film was small on nanometer order to maintain the microflow channel form after modification. Such findings will pave the way for the development of a new surface modification method for the PDMS microflow channel toward biomedical applications. 


\section{Acknowledgments}

This study was supported by research grants from Saitama Prefectural Academic-Industrial Research and Development Project Subsidy (Medical Innovetion) as "Development of Silicone Rubber (PDMS) Surface Modification Method toward Hydrophilic Surface" in FY2017-2018.

\section{References}

1 R. E. Holmlin, X. Chen, R. G. Chapman, S. Takayama, and G. M. Whiteside: Langmuir 17 (2001) 2841.

2 E. Ostuni, R. G. Chapman, M. N. Liang, G. Meluleni, G. Pier, D. E. Ingber, and G. M. Whitesides: Langmuir 17 (2001) 6336.

3 S. Chen, J. Zheng, L. Li, and S. Jiang: J. Am. Chem. Soc. 127 (2005) 14473.

4 Z. Zhang, M. Zhang, S. Chen, T. A. Horbert, B. D. Ratner, and S. Jiang: Biomaterials 29 (2008) 4285.

5 M. Tanaka, T. Sawaguchi, Y. Sato, K. Yoshioka, and O. Niwa: Tetrahedron Lett. 50 (2009) 4092.

6 M. Tanaka, T. Sawaguchi, Y. Hirata, O. Niwa, K. Tawa, C. Sasakawa, and K. Kuraoka: J. Colloid Interf. Sci. 497 (2017) 309.

7 S. Bauer, J. A. Finlay, I. Thomé, K. Nolte, S. C. Franco, E. Ralston, G. E. Swain, A. S. Clare, and A. Rosenhahn: Langmuir 32 (2016) 5663.

8 J. B. Schlenoff: Langmuir 30 (2014) 9625.

9 T. Wang, X. Wang, Y. Long, G. Liu, and G. Zhang: Langmuir 29 (2013) 6588.

10 T. Xiang, R. Wang, W. Zhao, S. Sun, and C. Zhao: Langmuir 30 (2014) 5115.

11 B. Yu, J. Zheng, Y. Chang, M. Sin, C. Chang, A. Higuchi, and Y. Sun: Langmuir 30 (2014) 7502.

12 P. Ren, H. Yang, H. Liang, X. Xu, L. Wan, and Z. Xu: Langmuir 31 (2015) 5851.

13 T. Wang, R. Kou, H. Liu, L. Liu, G. Zhang, and G. Liu: Langmuir 32 (2016) 2698.

14 Z. Zhang, M. Moxey, A. Alswieleh, A. J. Morse, A. L. Lewis, M. Geoghegan, and G. L. Leggett: Langmuir 32 (2016) 5048 .

15 L. Deng, M. Mrksich, and G. M. Whitesides: J. Am. Chem. Soc. 118 (1996) 5136.

16 R. G. Chapman, E. Ostuni, S. Takayama, R. E. Holmlin, L. Yan, and G. M. Whitesides: J. Am. Chem. Soc. $122(2000) 8303$.

17 E. Ostuni, R. G. Chapman, R. E. Holmlin, S. Takayama, and G. M. Whitesides: Langmuir 17 (2001) 5605.

18 R. S. Kane, P. Deschatelets, and G. M. Whitesides: Langmuir 19 (2003) 2388.

19 Y. Higaki, M. Kobayashi, D. Murakami, and A. Takahara: Polym. J. 48 (2016) 325.

20 M. Takai: Polym. J. (2018) https://doi.org/10.1038/s41428-018-0049-3

21 Y. Iwasaki and K. Ishihara: Anal. Bioanal. Chem. 381 (2005) 534.

22 J. Kuang and P. B. Messersmith: Langmuir 28 (2012) 7258.

23 M. Li, K. G. Neoh, L. Q. Xu, R. Wang, E. Kang, T. Lau, D. P. Olszyna, and E. Chiong: Langmuir 28 (2012) 16408.

24 S. Ye, Y. Jang, Y. Yun, V. Shankarraman, J. R. Woolley, Y. Hong, L. J. Gamble, K. Ishihara, and W. R. Wagner: Langmuir 29 (2013) 8320.

25 A. I. Barbosa and N. M. Reis: Analyst 142 (2017) 858.

26 M. Dawod, N. E. Arvin, and R. T. Kennedy: Analyst 142 (2017) 1847.

27 G. Z. Ou, X. J. Feng, W. Du, X. Liu, and B. F. Liu: Anal. Bioanal. Chem. 405 (2013) 7907.

28 H. Hillbog, J. F. Ankner, U. W. Gedde, G. D. Smith, H. K. Yasuda, and K. Wikstöm: Polymer 41 (2000) 6851.

29 J. Kim, M. K. Chaudhury, and M. J. Owen: J. Colloid Interf. Sci. 226 (2000) 231.

30 J. Kim, M. K. Chaudhury, M. J. Owen, and T. Orbeck: J. Colloid Interf. Sci. 244 (2001) 200.

31 H. Hillborg, N. Tomczak, A. Olàh, H. Schönherr, and G. J. Vancso: Langmuir 20 (2004) 785.

32 D. T. Eddington, J. P. Puccinelli, and D. J. Beebe: Sens. Actuators, B 114 (2006) 170.

33 H. Hillborg and U. W. Gedde: Polymer 39 (1998) 1991.

34 Y. Berdichevsky, J. Khandurina, A. Guttman, and Y.-H. Lo: Sens. Actuators, B 97 (2004) 402.

35 S. Hu, X. Ren, M. Bachman, C. E. Sims, G. P. Li, and N. Allbritton: Anal. Chem. 74 (2002) 4117.

36 S. Yeh, C. Chen, W. Chen, and C. Huang: Langmuir 30 (2014) 11386.

37 H. Katayama, Y. Ishihama, and N. Asakawa: Anal. Chem. 70 (1998) 2254.

38 H. Katayama, Y. Ishihama, and N. Asakawa: Anal. Chem. 70 (1998) 5272.

39 K. Kubiak, Z. Adamczyk, and M. Wasilewska: J. Colloid Interf. Sci. 457 (2015) 378.

40 T. Kunitake, A. Tsuge, and N. Nakashima: Chem. Lett. (1984) 1783. 
M. Shimomura and T. Kunitake: Thin Solid Films (1985) 243.

A. Durán-Álvarez, M. Maldonado-Domínguez, O. González-Antonio, C. Durán-Valencia, M. Romero-Ávila, F. Barragán-Aroche, and S. López-Ramírez: Langmuir 32 (2016) 2608.

A. Ge, M. Matsusaki, L. Qiao, M. Akashi, and S. Ye: Langmuir 32 (2016) 3803.

M. Tanaka and S. Kurosawa: J. Oleo Sci. 66 (2017) 699.

M. Tanaka, Y. Hirata, T. Sawaguchi, and S. Kurosawa: Arkivoc 2018 (2018) 330.

A. Mafi, D. Hu, and K. C. Chou: Langmuir 32 (2016) 10905.

H. Chen, C. Zhao, M. Zhang, Q. Chen, J. Ma, and J. Zheng: Langmuir 32 (2016) 3315.

M. Ishiyama, A. Shiga, K. Sasamoto, M. Minoguchi, and P. He: Chem. Pharm. Bull. 41 (1993) 1118

H. Tominaga, M. Ishiyama, F. Ohseto, K. Sasamoto, T. Hamamoto, K. Suzuki, and M. Watanabe: Anal. Commun. 36 (1999) 47.

\section{About the Authors}

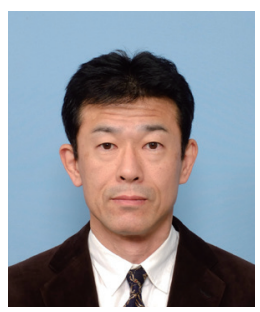

Mutsuo Tanaka received his B.S., M.S., and Ph.D. degrees from Osaka University, Japan, in 1985, 1987, and 1996, respectively. From 1989 to 2018, he was a researcher at the National Institute of Advanced Industrial Science and Technology (AIST). Since 2018, he has been a professor at Saitama Institute of Technology. His research interests are in material chemistry.

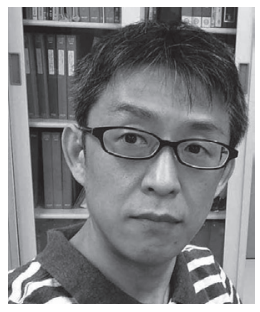

Yoshikatsu Ogawa received his B.S. degree from Tokyo University of Science, Japan, in 1991, M.S. degree from the University of Tsukuba, Japan, in 1993, and Ph.D. degree from Université de Strasbourg, France, in 2001. Since 1993, he has been a researcher at the National Institute of Advanced Industrial Science and Technology (AIST). His research interests are in chemical probes and cell biology.

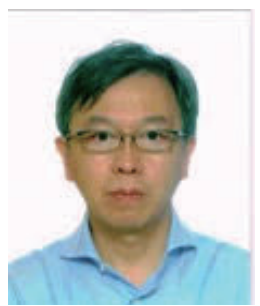

Yoshiki Hirata received his B.S. and M.S. degrees from Yamanashi University, Japan, in 1984 and 1986, respectively, and Ph.D. degree from Tokyo Institute of Technology, Japan, in 1990. Since 1990, he has been a researcher at the National Institute of Advanced Industrial Science and Technology (AIST). His research interests are in electrochemistry and surface chemistry.

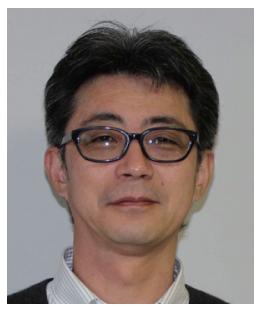

Takahiro Sawaguchi received his B.S., M.S., and Ph.D. degrees from Tohoku University, Japan, in 1985, 1988, and 1991, respectively. From 1992 to 1997, he was a researcher at Itaya Electrochemiscopy Project, ERATO/JST, Japan. Since 1998, he has been a researcher at the National Institute of Advanced Industrial Science and Technology (AIST). His research interests are in electrochemistry and surface science. 


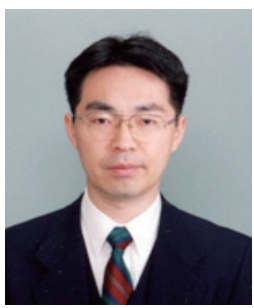

Shigeru Kurosawa received his B.E. and M.E. degrees from Nagaoka University of Technology in 1984 and 1986, respectively, and Ph.D. degree from Hokkaido University in 1990. Since 1990, he has been a researcher at the National Institute of Advanced Industrial Science and Technology (AIST). His research interests are in analytical chemistry. 